\title{
Genius and nonlinear dynamics
}

\section{Wlodzimierz Klonowski}

Correspondence:

wklon@ibib.waw.pl

Nalecz Institute of Biocybernetics and

Biomedical Engineering, Polish

Academy of Sciences, Warsaw, Poland

\begin{abstract}
Genius is defined as a person of outstanding mental skills. These skills result from genius' brain structure and physiology that are determined by genetics. It seems that environment and education may only inhibit development of a genius. Based on a simple model of brain phase space we formulate two hypotheses about dynamics of mental processes in genius' brain and the difference between genius thinking and a mental illness or insanity.
\end{abstract}

Keywords: Genius; Nonlinear dynamics; Deterministic chaos; Brain; Phase space; Attractor; Chaosensology; Conditional definition; Normality; Insanity

The soul needs more space than the body.

Axel Munthe (1857-1949)

Swedish writer, neurologist

Many concepts functioning in modern societies lack satisfactory, unambiguous definitions. Some definitions include conditions, for example in a form of evaluative adjectives such as 'outstanding ' or 'exceptional'. An object is called a work of art if it exerts exceptional (but otherwise quite subjective) emotional effect on the person who is watching this object. The same concerns the concept of genius. According to Merriam-Webster Dictionary genius is a 'person endowed with transcendent mental superiority, inventiveness, and ability (...like the Curies, Darwin, or Newton)' [1] and according to Advanced Learners Dictionary it is a 'very great and exceptional capacity of the mind or imagination' or a 'person having this capacity' [2].

Regular (unconditional) definition should contain an objective unambiguous description or specification, like 'Square is a quadrangle that has all right angles and all sides of equal length'. Regular conditional definition contains condition that may be ambiguous but is not evaluative, e.g. describes some activity that might take place, like for example Russian definition of 'nothing': 'NOTHING is half a liter of vodka for two persons, under condition that one of these persons does not drink'. Definition that contains evaluative condition, like for example an evaluative adjective, will be called evaluative conditional definition: 'A is $B$ under condition $c^{\prime}$ where the condition $c$ is subjective in the sense that it depends mainly on the experience of the evaluator. An object is a work of art under condition that the evaluator considers that it is exceptional, a person is a genius under condition that the evaluator considers him/her to be extremely gifted (artistically or mentally).

Of course, not every scientist is a genius. A co-discoverer of the helical structure of DNA, Nobel laureate James D. Watson (Nobel Prize in Physiology and Medicine in 1962 along with Francis Crick and Maurice Wilkins) has even stated very bluntly that vast majority of scientists is 'just stupid' [3].

(c) 2015 Klonowski. This is an Open Access article distributed under the terms of the Creative Commons Attribution License (http:// creativecommons.org/licenses/by/4.0), which permits unrestricted use, distribution, and reproduction in any medium, provided the original work is properly credited. 


\section{Genius, genetics, gender}

Nobody can learn how to be a genius - one has to be born a genius. It is obvious that nobody could teach Leonardo da Vinci how to construct flying machines. Famous Polish mathematician Hugo Steinhaus said about his former student and the 'greatest discovery' Stefan Banach who actually was considered a mathematical genius: "No one more than he [Banach] helped to dispel the harmful belief that in scientific competition lack of genius may be replaced by other merits." But there are scientists who say that apart from genetics genius depends also on 'other elements', such as intelligence and personality, and that social environment has also important influence. But somehow most of these other elements also depend almost exclusively on genetics. And the environment will not make a genius from a fool or even from a capable average person. Oppositely, environment may effectively inhibit the development of a person with a genetic predisposition to be a genius. Modern schools, universities and research centers through the educational systems without a doubt have wasted many potential geniuses.

The differences in the level of intelligence of individuals are also largely determined by differences in the genes. In the 90's of XX century American scientist Richard Plomin showed that the majority of children with very high IQ has 'intelligence gene' IGF2R that encodes a receptor protein for so called insulin-like growth factor 2, IGF2. In 2009 British scientists under the direction of Ian Deary demonstrated that human intelligence is rather polygenic (determined by many genes) [4]. But what factors other than genes determine intelligence? Another article co-authored by I.Deary shows that the conditions of family and school environment also play a crucial role in shaping person's intelligence, but at the same time do not change anything in terms of person's predisposition [5]. But even the influence of the environment can significantly depend on genetics. For example, breastfed babies may gain about 7 IQ points, but only if they have the appropriate version of the gene called FADS2; for 10 percent of children who have a mutated version of this gene the method of feeding has no effect on subsequent results of intelligence tests [6].

It has been shown experimentally that, at the genetic level, there exists a relationship between creativity and schizophrenia. The team led by Hungarian scientist Szabolcsa Kéri showed that the most creative persons (about 12 per cent of the examined group) have had two mutated copies of the gene encoding the protein called neuregulin 1; those with one copy of the mutated DNA fragment were also rated as being more creative than volunteers without the mutation. However, sometimes mutated neuregulin instead of enhancing creativity contributes to mental disorders [7]. The deciding factor may be just intelligence - in persons with a low intelligence the mutation may lead to psychiatric disorders. Already in 1883 Francis Galton said that intelligence is connected with effective operation of central nervous system and that this characteristic is an inherited feature.

Human physiology including sex is of course determined genetically. Psychophysiology introduced the concept of 'sex of the brain'. According to Schopenhauer psychophysiology of a genius (male) differs from that of an ordinary man; women according to Schopenhauer may be talented but their innate subjectivism causes that they cannot be geniuses. In Polish, and not only Polish, the word 'genius' is masculine and there is no feminine equivalent. But of course, Maria Sklodowska-Curie was undoubtedly a genius. Contemporary feminists claim that chaos theory and nonlinear dynamics in its essence exemplify the acceptance of feminist values, such as ambiguity and uncertainty, in contrast to the masculinist classical physics [8-10]. 


\section{Genius and madness in phase space}

The world in which we live is governed by the laws of nonlinear dynamics [11-15]. In modern physics dynamic system, regardless of the degree of complexity is described using a multi-dimensional phase space (state space), with coordinates uniquely characterizing the state of the system at any moment of time (i.e. each point of the phase space corresponds to certain state of the system under consideration), and the $d y$ namical rules (mappings) determining to what state the system will pass at the next moment of time $(t+1)$ from the given state at the moment $t$. In other words, the phase space is the set of all possible states of the dynamical system, and trajectories in phase space characterize processes, i.e. the evolution of the system over time. Nonlinear processes may exhibit the so-called deterministic chaos, that is an immense sensitivity to the initial conditions and parameters of the system.

Nonlinear dynamics - a branch of systems theory investigating the evolution of dynamical systems in which the result of action of a stimulus is not directly proportional to the strength of this stimulus - introduces the concept of attractor - subsets of the phase space to which the trajectories are proceeding as if they were attracted to. There are point attractors and attractors in the form of closed curves, so called limit cycles that correspond to (quasi)periodic processes. Attractors may be stable or unstable. The part of the phase space from which the trajectories proceeds to the attractor is called the basin of attraction of this attractor. In the case of a living organism stable attractors correspond to homeostatic states. Chaotic systems may posses so called strange attractors, dimension of which is not an integer number.

On Fig. 1 a simple example of attractors in two-dimensional phase subspace is shown. Unstable limit cycle 2 separates basins of attractions of the stable point attractor 0 and the basin of attraction of the stable limit cycle 1 . This means that if the system is in a state, that corresponds to the values of $\xi$ and $\eta$ lying within the area bounded by the dashed curve 2 then the following processes cause its returning to state 0 as shown by tapering spiral line with an arrow. If the system is in a state that corresponds to the

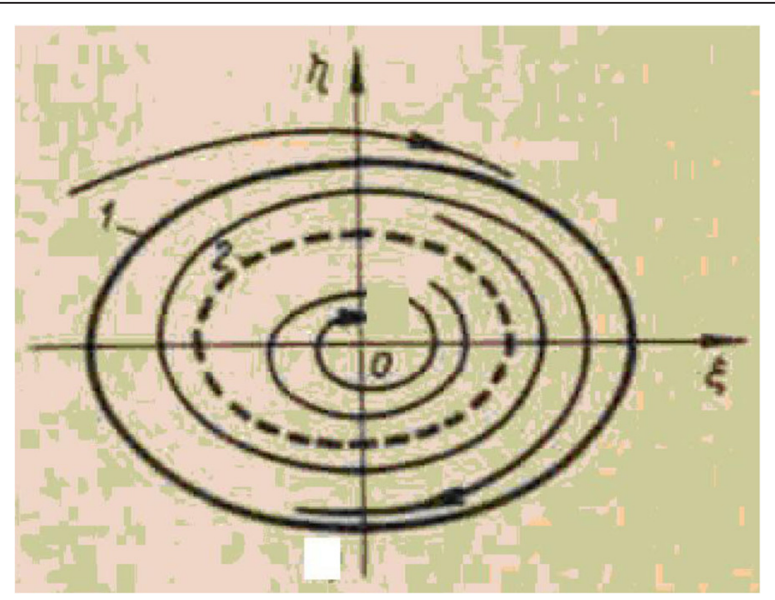

Fig. 1 Example of a two-dimensional phase space (described by two variables $\xi$ and $\eta$ ) of a dynamic system that has three attractors: stable point attractor (0); stable limit cycle (solid line, 1); unstable limit cycle (dashed line, 2). Unstable cycle 2 separates basins of attraction of stable attractors 0 and 1. Trajectories correspond to lines with arrows; the limit cycles 1 and 2 also constitute trajectories. This may represent a two-dimensional subspace of a multi-dimensional phase space 
values of $\xi$ and $\eta$ lying outside the area bounded by the dashed curve 2 then the following processes cause that the system tends to the limit cycle 1 as shown by the widening spiral line with an arrow. After reaching this limit cycle (any point on the curve 1) the system will be repeatedly passing in a specified direction through the states lying on this curve (in this example in a clockwise direction) until some fluctuation will not 'throw' the system out from this periodic attractor, e.g. to the basin of attraction of the point attractor 0 . If the system is in a state that corresponds to the values of $\xi$ and $\eta$ that lie outside the area bounded by the solid curve 1 then processes occurring in the system will cause the system to return to the limit cycle 1 (cf. trajectory with the arrow at the top of the Fig. 1).

The dynamics of a living organism can be described as a multi-dimensional trajectory in a phase space, whose axes correspond for example to the concentrations of various enzymes, the blood flow, the frequencies of meetings with other organisms of the same species and of other species, the intensity of different forms of behavior, etc. Such a space can be treated as composed of a certain minimal subspaces such that their further subdivision does not make sense and are only weakly related to each other [12-15].

Human brain cannot function in a completely deterministic way, because then we would not be able to create anything new, neither in a completely stochastic (random) way, because then we would not be able to perform any intended action, even to press a specific key on the keyboard with our finger. In creative processes human brain must therefore act as a complex dynamic system, showing among others features these of deterministic chaos.

We define the phase space of the brain, $\boldsymbol{B}$, as follows:

$$
B=(S, I, M, R, E, C, O)
$$

where $S$ is a set of variables characterizing self-awareness; $\boldsymbol{I}$ is a set of input variables, i.e. those determining effects of the environment on the brain and the body, such as impulses from sensory organs; $\boldsymbol{M}$ is a set of variables defining memory; $\boldsymbol{R}$ is the set of variables determining the so-called operational memory; $\boldsymbol{E}$ is a set of variables that define the emotional state; $\boldsymbol{C}$ is a set of variables that define the state of consciousness; $\boldsymbol{O}$ is the set of output variables, i.e. those determining effects exerted by the brain on effectors such as impulses send to the muscles. Contemporary knowledge of physiology and biochemistry of the brain is not enough to indicate what specific variables determine the phase space $\boldsymbol{B}$ and its subsets, in particular $\boldsymbol{S}, \boldsymbol{E}$, and $\boldsymbol{C}$. Such a subdivision of variables into several sets is just a matter of convenience. It is obvious that we will never be able even to enumerate all variables that 'define' brain's phase space, neither of course to measure them simultaneously.

While in the 'classical' theories of complex systems the state space itself does not change with time, our theory is a generalization of these theories - the processes occurring in the phase space of the brain $\boldsymbol{B}$ may change space $\boldsymbol{B}$ itself, at least temporarily, for example by making some of its areas inaccessible to other processes. One may find an analogue in lattice models of polymer solutions with excluded volume effect.

The rules that govern the dynamic changes of the brain have not yet been understood by science, and it is difficult to imagine that they ever will be explained in detail. The phase space of the brain has huge number of dimensions and only in very specific conditions the number of relevant dimensions can be reduced. The geometry of the phase 
space of the brain is of course not Euclidean - while some states of the brain may seem to be very different, the points corresponding to those states may be close to each other in phase space $\boldsymbol{B}$. Just like on the surface of the Earth at the so called line of date changing date Far East and Far West are adjacent one to another. In psychology there exists the effect of turning the feelings into its opposition, for example, love into hate. In the chaos of emotions [13] just a small change of 'initial conditions' can dramatically change emotions.

However, even a simple two-dimensional mathematical models allow physicists to formulate hypotheses about brain operation mechanisms, for example what are the differences between sub-consciousness and consciousness, between emotions (feelings) and logical thinking (thoughts), and that existence of a hypothetic Central Executive Structure in the brain is not necessary (cf. [13-15]). Using two-dimensional model shown in Fig. 1. I will try to explain my hypotheses concerning the essence of genius, and the difference between genius and insanity or mental illness.

On Fig. 1 a basin of attraction of a stable attractor $\boldsymbol{O}$ can be considered to correspond to 'mental health'. If any process, would it correspond to a thought or to a feeling, takes place it means that a phase trajectory has to come out from $\boldsymbol{O}$ that is the brain must have performed certain 'mental work'. But even if the trajectory is very complicated, after some time it returns to $\boldsymbol{O}$ - brain returns to the state of 'mental balance', 'state of rest'. However, if that trajectory goes so far that it crosses unstable limit cycle 2 the system no longer returns to a state $\boldsymbol{O}$ of 'mental balance'; instead the trajectory will be approaching closer and closer the limit cycle $\mathbf{1}$. The stable limit cycle and its basin of attraction can be considered to correspond to 'mental illness', while the unstable limit cycle 2 to a 'thin boundary line' between mental health and mental illness, while. If the brain reach a state that belongs to the stable limit cycle 1 it will be periodically passing again and again through the same states. Patients with obsessive-compulsive disorder are haunted by some obsessive thoughts and feelings again and again. 'Escape' from the basin of cycle 1 may require drastic measures, such as electroshocks. Of course, in the case of multi-dimensional phase space of the brain the phase space is much more complicated, instead of a point attractor $\boldsymbol{O}$ can be a whole compact set of such points or even a number of such sets, and cycles 1 and $\mathbf{2}$ can be replaced by complex multi-dimensional hypersurfaces.

But already based on the above two-dimensional model, we can formulate two important hypotheses about the genius:

\section{Hypothesis 1}

Genius is a person whose basin of attraction of the attractor 'mental health' $\boldsymbol{O}$ is exceptionally large, giving the genius 'open mind' without crossing the 'thin boundary line' of cycle 2 , that is, without falling into a mental illness. In most people, the basin of attraction of $\boldsymbol{O}$ is relatively small - famous Polish SF writer Stanislaw Lem coined the term Extensively mediocre minds [16].

\section{Hypothesis 2}

There are two kinds of geniuses. In some geniuses 'mental illness' limit cycle 1 is quite far away from the 'thin boundary line' of mental health 2 ; therefore, if this limit is exceeded genius simply becomes a mentally ill man. In other geniuses cycle 1 may be 
close to cycle 2 so the boundary of 'mental health' can be crossed relatively easily in both directions, as it really is the case of many brilliant artists, poets, and writers.

\section{Concluding remarks}

Although such mental qualities as a willingness to go beyond the clichés (common patterns) of thinking, the ability to create unconventional solutions, independence and nonconformity are often correlated with certain psychiatric disorders - insanity and genius are the two sides of the same coin. Arousal that foster creativity may occur in a human brain. However, most developers - in the broadest sense of the word - have been 'normal ' according to the ratings of their contemporaries, and most of the 'insane' have not been creative.

And here the question arises what does it really mean to be 'normal'? Normality is also relative. In the garden of a madhouse a patient with a fishing rod stays over a barrel of water; one doctor why passing by asks: "Are there really any fishes in this barrel?" and the patient answers: "In the barrel? Are you crazy, doctor?"

\section{Competing interests}

The author declares that he has no competing interests.

Received: 15 January 2015 Accepted: 15 June 2015

Published online: 12 August 2015

\section{References}

1. Webster's Third New International Dictionary of The English Language, Unabridged, Merriam-Webster Incorporated, Springfield, Mass., USA; Koenemann, Cologne, Germany, 1993.

2. The Advanced Learners Dictionary of Current English, $2^{\text {nd }}$ ed., Oxford University Press, London, 1963.

3. Watson JD. The Double Helix, George Weidenfeld Nicolson, 1968

4. Deary IJ, Johnson W, Houlihan LM. Genetic foundations of human intelligence. Hum Genet. 2009;126(1):215-32.

5. Davies $\mathrm{G}$ et al. Genome-wide association studies establish that human intelligence is highly heritable and polygenic. Molecular Psychiatry. 2011;16:996-1005.

6. Caspi A, et al. Moderation of breastfeeding effects on the IQ by genetic variation in fatty acid metabolism. PNAS. 2007;104(47):18860-5.

7. Kéri S. Genes for psychosis and creativity: a promoter polymorphism of the neuregulin 1 gene is related to creativity in people with high intellectual achievement. Psychological Science. 2009;20:1070.

8. Keller EF. Reflections on Gender and Science, Yale University Press, 1985

9. Haraway D. Situated Knowledges: The Science Question in Feminism and the Priviledge of Partial Perspective. Feminist Studies. 1988;14(Fall):579-90.

10. McClure MA. Feminism and Chaos Theory, in: Evan M. Berman, Ed. Encyclopedia of Public Administration and Public Policy Second Ed. 2011, ch. 151

11. Strogatz SH. Nonlinear Dynamics and Chaos: With Applications to Physics, Biology, Chemistry, and Engineering , 1994, Westview Press

12. Klonowski W. From conformons to human brains: an informal overview of nonlinear dynamics and its applications in biomedicine, Nonlinear Biomedical Physics, 1, 5 , 2007, BioMed Central, London, http:// www.nonlinearbiomedphys.com/content/1/1/5

13. Klonowski W. Nonlinear Dynamics in Psychophysiology - Feelings and Thoughts. In: Klonowski W, editor. Simplicity behind Complexity, Frontiers on Nonlinear Dynamics, vol. 3. Lengerich, Berlin: Pabst Science Publishers; 2004. p. 467-76.

14. Klonowski W. Consciousness, Subconsciousness, Biofeedback. Interdisciplinary Considerations with Medical Applications, in: Art and Science in Life Potential Development, M.Prstatic (Ed), Zagreb 2011, pp. 34-37

15. Klonowski W. Significance of time scale differences in psychophysics. Cognitive Processing. 2009;10(1):119-26.

16. Lem S. Flash (Okamgnienie, in Polish), Cracow, 2000 